\title{
Çocuk Hastalarda Kaudal Blokaj
}

\section{Caudal Blockage in Children}

\author{
Funda Durmaz
}

Anesteziyoloji ve Reanimasyon Bölümü, Tokat Devlet Hastanesi, Tokat, Türkiye

\section{öz}

Kaudal Anestezi tek başına en önemli pediatrik rejyonel anestezi tekniğidir. Kaudal anestezi, gerçekten öğrenmesi kolay, güvenliği kanıtlanmış ve birçok prosedür için kullanılabilen bir tekniktir.

Kaudal blok; umblikus altı girişimlerde kullanılabilen basit, güvenli ve etkili bir teknik olmayı sürdürmektedir.

Anahtar Kelimeler: kaudal anestezi, çocuk, ilaçlar, komplikasyon

\section{ABSTRACT}

Caudal Anesthesia is the most important pediatric regional anesthesia technique alone. Caudal anesthesia is a technique that is really easy to learn, safe to use and can be used for many procedures.

Caudal block; continues to be a simple, safe and effective technique that can be used in procedures under umbilicus

Keywords: caudal anesthesia, children, drugs, complication

Corresponding Author: Dr. Funda Durmaz

Address: Anesteziyoloji ve Reanimasyon Bölümü

Tokat Devlet Hastanesi, Tokat, Türkiye

Başvuru Tarihi/Received: 09-04-2018

E-mail: dfundadurmaz@gmail.com 


\section{GíRiş}

Kaudal Anestezi tek başına en önemli pediatrik rejyonel anestezi tekniğidir. Kaudal anestezi, gerçekten öğrenmesi kolay, güvenliği kanıtlanmış ve birçok prosedür için kullanılabilen bir tekniktir.

\section{Kaudal Blok}

Pediatrik hastalarda, postoperatif ağr1 hemodinamiyi olumsuz yönde etkilemekte ve konforu bozmaktadır. Campbell tarafindan 1933 yllında ilk kez uygulanan kaudal epidural analjezi pediatrik anestezide en popüler ve s1klikla tercih edilen rejyonel bloktur (1). Günümüzde kaudal blok, özellikle umbilikus altındaki cerrahi girişimlerde genel anestezi gereksinimini azaltmak ve postoperatif analjezi sağlamak amacı ile yaygın olarak kullanılmaktadır (2,3). Kaudal anestezi teknik olarak öğrenmesi kolay, güvenli ve birçok prosedür için kullanılabilen bir yöntemdir $(4,5)$.

\section{Anatomik İnceleme}

İnfantlarda dura va spinal kord spinal kanalda daha alt seviyelere ulaşır. (spinal kord doğumda L3, bir yaşında L1/L2 ve dura doğumda S4, bir yaşında S2) Küçük infantlarda dural kesenin sonu ile giriş yeri arasında sadece birkaç milimetrelik mesafe olabilir (6). Sakrum ve sakral hiatus son derece varyasyon gösteren anatomik yapılardır. Bununla birlikte; sakral hiatusun tepesi ve üst lateral sakral çıkıntıların arasında oluşan eşkenar üçgen, blok sırasında sakral hiatusun lokalizasyonunu belirlemede faydalidır (7). Okul öncesi çocuklarda gevşek olan epidural yağ tabakası lokal anesteziğin torasik alana yayılımını kolaylaştırır. Daha büyük çocuklarda (vücut ağırlı̆̆ $20 \mathrm{~kg}$ 'den fazla) torasik yayılımı sağlamak daha zordur ve kaudal blok genelikle ilk tercih değildir.
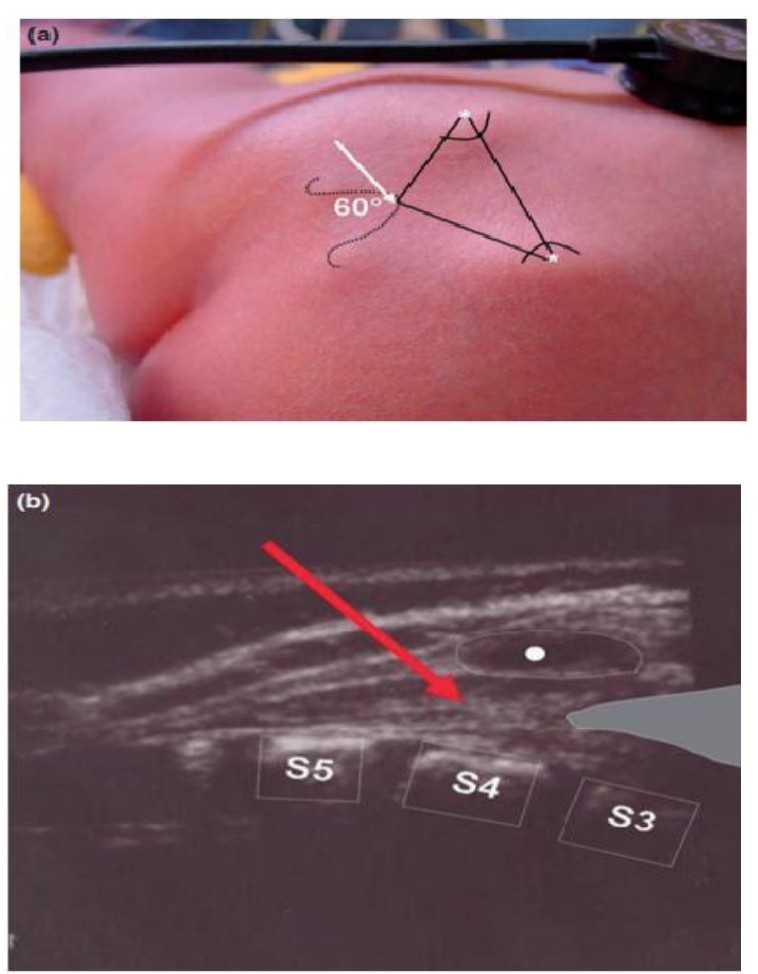

Şekil 1 sakral alanın anatomisi. (a) sakral hiatusa iğnenin giriş noktası. (b)dural sakın dar mesafesini gösteren sonografik görüntü.

\section{Hemodinamik Değişiklikler}

6-8 yaş aralığında yüksek spinal ve epidural blok düşük bazal sempatik ton nedeniyle minimal kardiyovasküler değişikliğe neden olmaktadır (8). Vücudun alt bölgelerindeki kan göllenmesi ve vazodilatasyon diğer vücut bölgelerindeki vazokonsantrasyon ile dengelenir. Bununla birlikte, neonatal ve küçük infantta kaudal ve genel anestezi kombinasyonu derin hipotansiyona neden olmaktadır.

İntratekal ve subdural epidural enjeksiyon genellikle total spinal anesteziyle birlikteyken, damar veya sakral kemiğe iğnenin yanlış yerleştirilmesi lokal anestetiğe bağlı sistemik toksisiteye neden olur. İğnenin intravasküler veya intratekal yanlış yerleştiğini göstermek için aspirasyon ile kan veya BOS'un geri gelmesi kesin ispat olmasina rağmen, negatif aspirasyon bu komplikasyonları önlemede yeterli değildir. Sistemik veya intratekal enjeksiyonu önlemenin en popüler metodu test dozu uygulamaktır. Bununla beraber bu yöntemin geçerliliği de hala literatürde 
sorgulanmaktadır ve birçok merkez bu uygulamayı terketmiştir (9). İntravasküler, intratekal veya subdural epidural iğne/kateter yanlış yerleşimini belirlemek için kullanılan klasik epidural test dozu ve diğer stratejiler bir review ile analiz edilmiştir (10). $0.5 \mu \mathrm{g} / \mathrm{ml}$ epinefrin enjeksiyonundan sonra sistolik arter basıncında $>15 \quad \mathrm{mmHg}$ basıç artışı görülmesinin sensitivitesinin yüksek olduğu gösterilmiştir. İntratekal ve subdural yanlış yerleşiminin kontrolü için daha iyi stratejilerin belirlenmesinde daha çok çalışmaya ihtiyaç vardır.

\section{İlaçlar ve Dozlar}

\section{Lokal Anestezikler}

Ropivakain \%0.2 çocuklarda kaudal anestezi için uygundur. Bupivakain ile karşılaştırıldığında benzer analjezi süresi sağlar fakat motor blokaj insidansı düşüktür. Ciddi toksisite azdır ve masif intravasküler enjeksiyona bağlıdır. Bupivakainin \%0.125'lik konsantrasyonu motor blokaja neden olmadan postoperatif ağrı kontrolü sağlamıştır (11). Biraz yüksek konsantrasyonları cerrahi sırasında daha hafif bir genel anestezi uygulanmasını sağlayabilir (12). Pratikte kaudal blok uygulanacak bir infant için 1-1.2 $\mathrm{mg} / \mathrm{kg} \% 0.125$ lik bupivakain epinefrin ile birlikte veya $\% 0.125$ lik bupivakain ile $\% 1$ lik prilokainin 1:1 karışımı kullanılır. Kabaca $1 \mathrm{mg} / \mathrm{kg}$ birçok vaka için uygundur. Lokal anestezik hacmi genellikle $25 \mathrm{ml}$ ile kısıtlanır. Son zamanlarda birçok girişim ile anatomik yayılım x-ray ya da ultrason kullanılarak gözlenmektedir(13,14,15). Bununla birlikte, blokajın klinik gözlemi ile anatomik yayılım arasında zayıf bir korelasyon vardır.

Bupivakain, levobupivakain ve ropivakain uygun lokal anesteziklerdir. Tüm lokal anestezikler pediatrik anestezi için güvenilir bulunur, Amerikan Kardiyak Arrest Topluluğu 343 vakanın sadece 7 tanesinde lokal anesteziğin kazayla uygulanması nedeni ile oluşan arrest bildirmişlerdir $(16,17)$.

\section{Adjuvanlar}

Adjuvanlar analjezi süresini uzatmak için kullanılır $(18,19)$. Epinefrin $5(\mu \mathrm{g} / \mathrm{ml})$ infantta kaudal epidural bupivakainin etkisini uzatmaktadır ve intravasküler enjeksiyonu önler ve en azından test dozunda kullanılmalıdır (20). Epinefrinsiz lokal anestezik güvenilir EKG değişikliklerine neden olmaz. Pratikte $0.5 \mu \mathrm{g} / \mathrm{ml}$ epinefrin $0.1-0.2$ $\mathrm{mg} / \mathrm{kg}$ lokal anestezik solüsyonuna katılır.

1-2 $\mu \mathrm{g} / \mathrm{ml}$ klonidin eklenmesinin kaudal blok sonrası analjezi süresini uzattığ 1 gösterilmiştir. Bu dozda yan etkiler minimaldir fakat yüksek dozda örneğin $5 \mu \mathrm{g} / \mathrm{ml}$ gibi, sedasyon, hipotansiyon ve bradikardi görülür (21). Küçük infantlarda postoperatif apne görülebileceği için klonidin kullanılmamalıdır (22-25). Pratikte klonidin sedatif etkileri uzattığı için kullanılmamaktadır.

Morfin mükemmel uzun süreli analjezi sağlar(26). Bununla birlikte yan etkileri yaygındır, bulantı, üriner retansiyon, kaşıntı ve solunum depresyonu gibi (27). Sonuç olarak kaudal opioidlerin rolü sorgulanmaktadır (28). Birkaç istisnai vaka dışında risk-yarar analizi morfin kullanımını belirler. Kaudal kateter uygulanan bir vakada optimal tek doz 33-50 $\mu \mathrm{g} / \mathrm{ml}$ olarak gösterilmiştir (29).

Daha fazla lipofilik olan opioidler; fentanil (30), sufentanil (31), petidin (32), diamorfin (33) veya tramadol (34) yan etkilere sahiptir ve analjezi süresini kliniğe uygun olarak uzatmaz. Kaudal bupivakaine eklenen ketamin analjezi süresini yaklaşık olarak üç misli artırır ve optimal doz $0.5 \mathrm{mg} / \mathrm{kg}$ 'dir. S-ketamin ile de güzel sonuçlar alınmaktadır. Bununla birlikte; neostigmin veya midazolama benzer şekilde potansiyel nörotoksisite bir problemdir. $\mathrm{Bu}$ durum s-ketamin kullanımını engeller(35).

\section{Komplikasyonlar}

Kaudal blok için komplikasyon oranı 0.7/1000'dir. Tablo 1'de komplikasyonlar özetlenmiştir. 
Tablo 1- Kaudal blokaj komplikasyon ve sonuçları

\begin{tabular}{|l|l|}
\hline Komplikasyon & Sonuç \\
\hline Dural delinme & Total spinal anestezi \\
Antravasküler enjeksiyon & Konvülsiyon, aritmi \\
Rektal penetrasyon & - \\
illaç overdozu & - \\
Morfin apnesi & Aritmi \\
Deri lezyonu & Apne \\
\hline
\end{tabular}

Spinal kordun aşağı yerleşmiş olması ve nöroenterik kistler gibi anomaliler kaudal anestezi esnasında iğnenin intratekal alana yerleşme riskini artırmaktadır. Bu defektlerden bazıları tanı almamış olabilir. Semptomlar alt ekstremite güçsüzlüğü ve inkontinans şeklinde ortaya çıabilir. Lumbosakral spinal bozukluk olan hastaların \%50-100'ünde gamzeleşme, k1llanma, hemanjiom ve lipomdan nevüs, kırmızı şarap lekesi ve hiperpigmentasyona uzanan kutanöz anormallikler görülebilir (36).

\section{Diğer rejyonel tekniklerin kaudal blok ile karşılaştırılması}

\section{Lumbar ve transsakral epidural analjezi}

Lumbar epidural anesteziyi özellikle L4/5 veya $\mathrm{L} 5 / \mathrm{S} 1$ seviyesinden uygulamak oldukça kolaydır. Epidural aralık hava ya da salin ile direnç kaybı yöntemi ile doğrulanır. Bununla birlikte; epidural alana hava verilmemesi için dikkatli olunmalıdır. Küçük infantta fazla miktarda hava verilmesi komplikasyonlara neden olur (37). Yan etkiler ve teknik problemler oluşabilir, yine de epidural enjeksiyonun riski daha azdır. Kaudal enjeksiyonla karşılaştırıldığında; paraplejiyi de içeren nörolojik komplikasyonlar lumbar yaklaşımda bildirilmiştir (38-40). Abdominal ve bazen torasik işlemlerde epidural kateterler tek başına lokal anestezik kullanımı ile analjezi sağlamada yetersizdir. Pediatrik hastalarda, transsakral yaklaşım uygulanabilir fakat dural ponksiyon riski fazladır ve L5/S1 yaklaşımdan farklı bir avantaj1 yoktur.

\section{İlioingüinal sinir bloğu , penil blok}

20-25 kg üzeri çocuklarda, ilioingüinal sinir bloğu ingüinal insizyon sonrasındaki ağrının önlenmesinde kaudal anesteziye alternatif olarak siklıkla kullanılmaktadır. Fakat bu teknik kaudal anestezi ile karşılaştırıldığında daha az efektiftir ve tam bir analjezi sağlamaz üstelik ultrason altında yapılmayan kör teknikte intestinal ponksiyon ve femoral sinir bloğu gibi riskler taşır (41). Tipik olarak hızlı absorbsiyon ile yüksek plazma seviyeleri oluşur (42). Penil cerrahi için, cerrahi bölgesinde uzun süreli analjezi sağladığ daha az komplikasyona yol açtığ blok avantajlıdır $(43,44)$. Hipospadias onarımı gibi geniş cerrahi prosedürlerde kaudal anestezi ve penil blok kombine edilmektedir.

\section{Sonuç}

Kaudal blok; umblikus altı girişimlerde kullanılabilen basit, güvenli ve etkili bir teknik olmayı sürdürmektedir.

\section{Kaynaklar}

1. Campbell MF. Caudal anesthesia in children. Am J Urol 1933; 30:245-249.

2. Tyler DC. Pharmacology of pain management. Pediatr Clin North Am. 1994; 44(1) 59-71.

3. Brill JE. Control of pain.. Crit Care Clin. 1992; 8(1): 203-18.

4. Schuepfer G, Konrad C, Schmeck J et al. Generating a learning curve for pediatric caudal epidural blocks: an empirical evaluation of technical skills in novice and experienced anesthetists. Reg Anesth Pain Med 2000; 25: 385388

5. Gunter J. Caudal anesthesia in children: a survey. Anesthesiology 1991; 75: A936.

6. Adewale L, Dearlove O, Wilson B et al. The caudal canal in children: a study using magnetic resonance imaging. Pediatr Anesth 2000; 10: 137-141.

7. Senoglu N, Senoglu M,Oksuz H, Gumusalan Y, Yuksel KZ, Zencirci B et al. Landmarks of the sacral hiatus for caudal epidural block: an anatomical study. $\mathrm{Br} \mathrm{J}$ Anaesth 2005;95:692-5.

8. Flandin-Ble'ty C, Barrier G. Accidents following extradural analgesia in children. The results of a retrospective study. Pediatr Anesth 1995; 5: 41-46. 
9. Tobias JD. Caudal epidural block: a review of test dosing and recognition of systemic injection in children. Anesth Analg 2001;93:1156-61

10. Guay J. The epidural test dose: a review. Anesth Analg 2006;102:921-9.

11. Wolf AR, Valley RD, Fear DW et al. Bupivacaine for caudal analgesia in infants and children: the optimal effective concentration. Anesthesiology 1988; 69: 102-106.

12. Gunter JB, Dunn CM, Bennie JB et al. Optimum concentration of bupivacaine for combined caudal-general anesthesia in children. Anesthesiology 1991; 75: 57-61.

13. Koo BN, Hong JY, Kil HK. Spread of ropivacaine by a weight-based formula in a pediatric caudal block: a fluoroscopic examination. Acta Anaesthesiol Scand 2010; 54: $562-565$

14. Thomas ML, Roebuck D, Yule C et al. The effect of volume of local anesthetic on the anatomic spread of caudal block in children aged 1-7 years. Pediatr Anesth 2010; 20: 1017-1021.

15. Lundblad M, Lonnqvist PA, Eksborg $\mathrm{S}$ et al. Segmental distribution of high-volume caudal anesthesia in neonates, infants, and toddlers as assessed by ultrasonography. Pediatr Anesth 2011; 21: 121-127.

16. Bhananker SM, Ramamoorthy C, Geiduschek JM et al. Anesthesia-related cardiac arrest in children: update from the Pediatric Perioperative Cardiac Arrest Registry. Anesth Analg 2007; 105: 344-350.

17. Morray JP, Geiduschek JM, Ramamoorthy C et al. Anesthesia-related cardiac arrest in children: initial findings of the Pediatric Perioperative Cardiac Arrest (POCA) Registry. Anesthesiology 2000; 93: 6-14

18. Ansermino M, Basu R, Vandebeek $\mathrm{C}$ et al. Nonopioid additives to local anaesthetics for caudal blockade in children: a systematic review. Pediatr Anesth 2003; 13: 561-573.

19. de Beer DA, Thomas ML. Caudal additives in children-solutions or problems? Br J Anaesth 2003; 90: 487498.

20. Veyckemans F, Van Obbergh LJ, Gouverneur JM. Lessons from 1100 pediatric caudal blocks in a teaching hospital. Reg Anesth 1992; 17: 119-125.

21. Mauch J, Kutter AP, Madjdpour $\mathrm{C}$ et al. Electrocardiographic alterations during intravascular application of three different test doses of bupivacaine and epinephrine: experimental study in neonatal pigs. Br J Anaesth 2010; 104: 94-97.

22. Lee JJ, Rubin AP. Comparison of a bupivacaineclonidine mixture with plain bupivacaine for caudal analgesia in children. Br J Anaesth 1994; 72: 258-262.

23. Motsch J, Bo" ttiger BW, Bach A et al. Caudal clonidine and bupivacaine for combined epidural and general anaesthesia in children. Acta Anaesthesiol Scand 1997; 41: 877883.

24. Breschan C, Krumpholz R, Likar R et al. Can a dose of 2 microg. $\mathrm{kg}(-1)$ caudal clonidine cause respiratory depression in neonates? Pediatr Anesth 1999; 9: 81-83.

25. Bouchut JC, Dubois R, Godard J. Clonidine in preterm-infant caudal anesthesia may be responsible for postoperative apnea. Reg Anesth Pain Med 2001; 26: 83-85.

26. Fellmann C, Gerber AC, Weiss M. Apnoea in a former preterm infant after caudal bupivacaine with clonidine for inguinal herniorrhaphy. Pediatr Anesth 2002; 12: 637-640.

27. Galante D. Preoperative apnea in a preterm infant after caudal block with ropivacaine and clonidine. Pediatr Anesth 2005; 15: 708-709.

28. Krane EJ, Jacobson LE, Lynn AM et al. Caudal morphine for postoperative analgesia in children: a comparison with caudal bupivacaine and intravenous morphine. Anesth Analg 1987; 66: 647-653.

29. Krane EJ. Delayed respiratory depression in a child after caudal epidural morphine. Anesth Analg 1988; 67: 79-82.

30. Lonnqvist PA, Ivani G, Moriarty T. Use of caudalepidural opioids in children: still state of the art or the beginning of the end? Pediatr Anesth 2002; 12: 747-749.

31. Erol A, Tavlan A, Tuncer $S$ et al. Caudal anesthesia for minor subumbilical pediatric surgery: a comparison of levobupivacaine alone and levobupivacaine plus sufentanil. J Clin Anesth 2008; 20: 442-446.

32. Kumar TPS, Jacob R. A comparison of caudal epidural bupivacaine with adrenaline and bupivacaine with adrenaline and pethidine for operative and postoperative analgesia in infants and children. Anaesth Intensive Care 1993; 21: $424-428$.

33. Kelleher AA, Black A, Penman S et al. Comparison of caudal bupivacaine and diamorphine with caudal bupivacaine alone for repair of hypospadias. Br J Anaesth 1996; 77: 586590.

34. Prosser DP, Davis A, Booker PD et al. Caudal tramadol for postoperative analgesia in paediatric hypospadias surgery. Br J Anaesth 1997; 79: 293-296.

35. Cook B, Doyle E. The use of additives to local anaesthetic solutions for caudal epidural blockade. Pediatr Anesth 1996; 6: 353-359.

36. Flandin-Ble'ty C, Barrier G. Accidents following extradural analgesia in children. The

results of a retrospective study. Pediatr Anesth 1995; 5: 4146.

37. Ecoffey C, Samii K. Neurologic complication after epidural anesthesia in a 15-yearold boy. Ann Fr Anesth Re'anim 1990; 9:398.

38. Zeidan A, Narchi P, Goujard E et al. Postoperative nerve irritation syndrome after epidural analgesia in a six-yearold child. Br J Anaesth 2004; 92: 146-148

39. Breschan C, Krumpholz R, Jost R et al. Intraspinal haematoma following lumbar epidural anaesthesia in a neonate. Pediatr Anesth 2001; 11: 105-108.

40. Jo ${ }^{*}$ hr M, Sossai R. Colonic puncture during ilioinguinal nerve block in a child. Anesth Analg 1999; 88: 1051-1052.

41. Amory C, Mariscal A, Guyot $\mathrm{E}$ et al. Is ilioinguinal/iliohypogastric nerve block always totally safe in children? Pediatr Anesth 2003;13: 164-166.

42. Frigon C, Mai R, Valois-Gomez $\mathrm{T}$ et al. Bowel hematoma following an iliohypogastric-ilioinguinal nerve block. Pediatr Anesth 2006; 16: 993-996.

43. Lipp AK, Woodcock J, Hensman B et al. Leg weakness is a complication of ilio-inguinal nerve block in children. Br J Anaesth 2004; 92: 273-274.

44. Smith T, Moratin P, Wulf H. Smaller children have greater bupivacaine plasma concentrations after ilioinguinal block. Br J Anaesth 1996; 76: 452-455. 